\title{
Implementación del procedimiento para el control del producto no conforme en una planta torrefactora de café"
}

\author{
Implementation of a Non-Conforming Product Control \\ System in a Roaster Factory
}

Recibido: 18 de junio del 2009 Revisado: 11 de septiembre del 2009 Aceptado: 30 de septiembre del 2009

\begin{abstract}
Germán Eduardo Galindo Ospina*
José Omar Henao Uribe

Oscar Rujana Quintero

Convenio USTA-ICONTEC, Bogotá, Colombia

\section{Resumen}

Este artículo presenta la implementación del procedimiento para el control del producto no conforme a lo largo del proceso de transformación de café verde a café tostado en grano o molido, que se lleva a cabo en la planta torrefactora. Se describe la metodología a seguir para identificar, controlar y dar tratamiento a los productos que no son conformes, es decir, que

\footnotetext{
* Artículo de investigación aplicada.

** Correspondencia: Germán Eduardo Galindo Ospina. Especialista en Administración y Gerencia de Sistemas de la Calidad. Convenio USTA-ICONTEC.Correo electrónico: gegojo@hotmail.com José Omar Henao Uribe. Especialista en Administración y Gerencia de Sistemas de la Calidad. Convenio USTA-ICONTEC. Correo electrónico: joshenao@gmail.com

Oscar Rujana Quintero. Especialista en Administración y Gerencia de Sistemas de la Calidad. Convenio USTA-ICONTEC. Correo
} electrónico: orujana@gmail.com
\end{abstract}

no se ajustan a los requisitos establecidos en las caracterizaciones de productos del Sistema de Gestión de Calidad.

Palabras clave: criterios, inspección, producto no conforme, torrefactora. sistema de gestión de la calidad.

\begin{abstract}
Implementation of the procedure for controlling nonconforming product during the processing of green coffee to roasted coffee beans in the roasters factory, describing the methodology followed to identify, monitor and provide treatment to non-conforming products with the characterizations requirements according to the Quality Management System.
\end{abstract}

Key words: criteria, inspection, non-conforming product, roaster, quality management system. 


\section{INTRODUCCIÓN}

Para cualquier organización recibir quejas o reclamos es un evento que genera impacto. Para el caso de las industrias manufactureras un reclamo relacionado con el producto implica fallas en los procesos, en la inspección o en el control del producto no conforme.

En un proceso de certificación, cuando el adecuado control de los procesos demanda tiempo y recursos es muy conveniente iniciar la implementación con el procedimiento para el control del producto no conforme. Definir pautas para identificar, controlar y prevenir el uso inadecuado de aquellos productos defectuosos asegura de manera casi inmediata que estos difícilmente llegarán al cliente final.

Es probable que en los primeros meses de ejecución se evidencien fallas en la organización, que manejadas de manera adecuada se pueden convertir en oportunidades de mejora. Las distintas opciones de tratamiento de los productos no conformes, como el reproceso y la adecuación, por sí solas no aportan a la mejora, es necesario realizar un análisis de causas que permita identificar el origen de las fallas y de esta manera poder corregirlas.

En este trabajo se muestran tres casos representativos de la detección de lotes defectuosos, que dieron inicio a los procedimientos de control de producto no conforme y de acciones correctivas. Esta dupla de procedimientos permitió detectar y corregir las causas de los defectos para cada caso.

Los resultados para la organización son evidentes y la implementación de este procedimiento genera la confianza suficiente en los Sistemas de gestión de la calidad, para que una de las metas del plan estratégico a mediano plazo sea la certificación bajo los criterios de la norma NTC-ISO 9001:2008

\section{METODOLOGÍA}

La primera etapa consistió en un conocimiento de la organización y de los procesos que allí se manejan, luego se realizó un diagnóstico del estado de la organización frente a los requisitos de la norma NTC-ISO 9001. Teniendo en cuenta el tiempo disponible, los esfuerzos se concentraron en implementar un procedimiento para el cumplimiento del numeral 8.3 de la norma NTC-ISO 9001.

Al tener el mapa de procesos suministrado por la organización se realizó el flujograma del proceso de transformación de café verde a café tostado en grano 0 molido, en él se identificaron los distintos puntos de control. Para que el flujograma fuera funcional, fue necesario observar detenidamente los procesos e identificar los sitios donde se realiza algún tipo de inspección o donde hay puntos críticos en los cuales seria necesario implementar una inspección adicional.

A continuación se implementó la inspección de aceptación para la materia prima, los insumos y el producto procesado. Elaborar un plan de muestreo implicó conocer el nivel de calidad requerido por el consumidor final.

Teniendo los controles definidos, se realizó la elaboración de las fichas técnicas de los distintos productos elaborados en la planta torrefactora, los cuales fijan los parámetros para la aceptación o rechazo de los productos. Para cada característica a evaluar se determinó la criticidad de su eventual incumplimiento, lo que permite dar un tratamiento adecuado al producto no conforme según sea el caso.

Luego, los resultados logrados después de dos meses de implementación del procedimiento desarrollado se muestran resumidos en cuatro casos representativos de productos no conformes. Su identificación, tratamiento y acciones de mejora fueron desarrolladas para cada caso. 
Por último se hace un pequeño recuento de los beneficios obtenidos y lecciones aprendidas, tanto en el nivel personal como en el organizacional.

\section{Diagnóstico}

Para conocer el estado inicial de la organización frente a la norma ISO 9001, fue necesario realizar un diagnóstico de todos los numerales de la norma. Para ilustrar el objetivo principal de este trabajo, se hizo énfasis en el numeral 8.3 de la norma control de producto y/o servicio no conforme.

\section{Flujograma}

Con el fin de identificar los puntos críticos se realizó un flujograma de las actividades desarrolladas durante el proceso de transformación del café verde a café tostado en grano o molido.

La literatura existente referente al proceso de transformación de café verde en café procesado es bastante limitada y pocas veces describe el manejo de un producto que no cumpla con las especificaciones del diseño. Los distintos autores se enfocan en la importancia de un café bien tostado, con un grado óptimo de molienda y una adecuada preservación del producto (Beattie, 2009; Davids, 2003; Ephraim, 2009). El proceso de transformación está compuesto por las siguientes etapas:

- Recepción de café verde

- Tostión

- Mezcla

- Molienda

- Empaque

- Producto terminado

\section{Identificación de puntos críticos}

Con base en el flujograma elaborado se realiza un estudio detallado de cada uno de los puntos críticos, de la inspección que se efectúa en cada uno de ellos, de las características a evaluar, del producto no Conforme que se pueda identificar y del tratamiento para cada caso.

Punto 1. Recepción de café verde: Siendo el café excelso la materia prima más importante en nuestro proceso, se realiza toma de muestras al $100 \%$ de los sacos ingresados a la planta torrefactora.

Punto 2. Grado de tostión del café: El punto de tueste del café afecta en gran medida la calidad de la bebida final. Para controlar este aspecto se realiza una medición del color del café tostado.

Punto 3. Grano partido: Si el café a empacar es café tostado y en grano, se toma muestra del café a la salida del homogeneizador y es llevada al laboratorio de calidades

Punto 4. Granulometría: Es una variable determinante para que el producto final cumpla su función, pues la granulometría influye directamente sobre los distintos mecanismos de extracción liquido - sólido.

Punto 5. Auto inspección en el empaque: Tanto en la línea de empaque automático como en la de empaque manual, hay características a evaluar que afectan la conformidad del producto. Básicamente son:

- Hermeticidad

- Estado de la válvula

- Peso

- Dimensiones

- Arrugas

- Rotulado

Punto 6. (Implementado). Inspección en del producto final: El lote de producto terminado debe estar plenamente empacado e identificado. Una vez listo se informa al área de calidades y se procede a realizar la inspección de aprobación. 
Este control no se realizaba, por lo que fue necesario implementar métodos de inspección y criterios de aceptación para lotes de producto terminado.

\section{Implementación gráficos de control}

La implementación de gráficos de control para los procesos de tostión y de molienda ha demostrado que estos procesos no se encuentran bajo control estadístico. Tener puntos muestrales por fuera de los límites de control superior e inferior indica que hay una serie de causas asignables que necesitan ser corregidas.

La primera etapa se basa en hallar las causas de estas variaciones utilizando diagramas de causa efecto. El identificar el origen de estas variaciones ha permitido tomar una serie de decisiones referentes a las causas que tienen mayor impacto sobre el problema (Juran \& Blanton, 2001).

\section{Implementación del muestreo de aceptación}

El muestreo de aceptación se ocupa de la inspección y la toma de decisiones respecto al producto terminado, que a pesar de ser uno de los aspectos más antiguos del aseguramiento de la calidad no estaba implementado. El muestreo de aceptación también se implementó para la recepción de materia prima e insumos (Montgomery, 2004).

Implementar el muestreo de aceptación ha generado un historial de calidad del producto terminado, el cual no existía hasta que se inició este proyecto. Esto ha permitido una retroalimentación directa a los demás procesos y ha ayudado a determinar qué controles están siendo adecuados o no. Hacer el muestreo ejerce presión sobre el colectivo de la planta para que mejoren el proceso de producción.

\section{Implementación de criterios de aceptación o rechazo}

Para medir la calidad y conformidad del café procesado es conveniente detallar las distintas dimensiones de calidad:

- Desempeño

- Confiabilidad

- Durabilidad

- Estética

- Características incluida

- Calidad percibida

- Conformidad con los estándares

\section{Elaboración de fichas técnicas}

Para poder identificar un producto como no conforme es necesario comparar las variables a evaluar con los criterios de aprobación o rechazo definidos para esta variable. Al no existir estos "limites" es prácticamente imposible saber cuándo un producto es defectuoso. Para tener un compendio de variables y sus límites, fue necesario elaborar fichas técnicas para cada una de las doce presentaciones distintas de los productos elaborados en la planta torrefactora.

Al realizar la inspección y evaluación de los lotes de producto terminado, los resultados son comparados con los criterios relacionados en la ficha técnica de cada producto y, con base en su cumplimiento, emitir el concepto de aprobación o rechazo.

\section{Manejo de producto no conforme}

Después de haber recorrido las etapas de diagnóstico, flujograma, identificación de puntos críticos, gráficos de control, muestreo, criterios de captación, y la elaboración de fichas técnicas para cada producto es posible identificar los productos no conformes al final y durante el proceso de transformación de café excelso a café tostado en grano o molido. 
Identificar un producto que no cumpla con las especificaciones de calidad previamente establecidas permite caracterizar el producto, evaluar la criticidad y de esta manera dar una manejo adecuado para cada una de las no conformidades.

Tabla 1. Manejo definido para cada una de las no conformidades, según su criticidad. Fuente: los autores.

\begin{tabular}{|c|c|c|c|}
\hline CARACTERÍSTICA NO CONFORME & NO CONFORMIDAD & CRITICIDAD & TRATAMIENTO \\
\hline & Defecto en taza & Crítico & Se desecha y pasa a compost \\
\hline Análisis sensorial & $\begin{array}{l}\text { No cumple el perfil de taza, } \\
\text { atributos esperados }\end{array}$ & $\begin{array}{l}\text { Mayor A } \\
\text { Mayor B }\end{array}$ & $\begin{array}{c}\text { - El laboratorio determina su } \\
\text { utilización final } \\
\text { - Libera el producto } \\
\text { - Se dona }\end{array}$ \\
\hline Grado de tostación & $\begin{array}{l}\text { El punto de tostión es más claro } \\
\text { u oscuro que lo especificado }\end{array}$ & $\begin{array}{l}\text { Mayor A } \\
\text { Mayor B }\end{array}$ & $\begin{array}{l}\text { - El laboratorio determina su } \\
\text { utilización final } \\
\text { - Libera el producto } \\
\text { - Se desecha }\end{array}$ \\
\hline Granulometría & $\begin{array}{l}\text { El punto de tostión es más claro } \\
\text { u oscuro que lo especificado }\end{array}$ & $\begin{array}{l}\text { Mayor A } \\
\text { Mayor B }\end{array}$ & $\begin{array}{l}\text { - El laboratorio determina su } \\
\text { utilización final } \\
\text { - Libera el producto } \\
\text { - Se dona }\end{array}$ \\
\hline Humedad $(\% \mathrm{~m} / \mathrm{m})$ & El $\%$ de humedad excede el $5 \%$ & Mayor A & -Se dona \\
\hline \% Oxígeno & $\begin{array}{l}\text { El\% de oxígeno excede el } \\
\text { limite }\end{array}$ & Mayor B & -Se reprocesa \\
\hline \multirow{5}{*}{ Empaque } & El empaque no es hermético. & Mayor A & -Se reprocesa \\
\hline & La bolsa no es simétrica & $\begin{array}{c}\text { Mayor B } \\
\text { Menor }\end{array}$ & $\begin{array}{c}\text { - Se reprocesa } \\
\text { - Libera el producto } \\
\end{array}$ \\
\hline & $\begin{array}{l}\text { Las fechas de vencimiento no } \\
\text { corresponden }\end{array}$ & $\begin{array}{l}\text { Mayor B } \\
\text { Menor }\end{array}$ & $\begin{array}{c}\text {-Se reprocesa } \\
\text { - Libera el producto }\end{array}$ \\
\hline & El laminado no corresponde & Mayor A & -Se reprocesa \\
\hline & Peso fuera de especificaciones & Mayor B & $\begin{array}{c}\text {-Se reprocesa } \\
\text {-Libera el producto }\end{array}$ \\
\hline Recuento de aeróbios mesófilos & \multirow{3}{*}{ El resultado excede los limites } & \multirow{3}{*}{ Crítico } & \multirow{3}{*}{ Se desecha y pasa a compost } \\
\hline NMP de coliformes & & & \\
\hline NMP de coliformes fecales & & & \\
\hline
\end{tabular}

\section{ANÁLISIS}

La metodología aplicada permitió tener un conocimiento claro del proceso industrial de la transformación del café excelso a tostado en grano o molido. Identificar y caracterizar los controles realizados en las distintas etapas del proceso ha permitido hacer un cuidadoso seguimiento al proceso que sigue el producto.

\section{RESULTADOS}

Poder identificar un producto no conforme en la planta torrefactora permite evitar que productos defectuosos lleguen al cliente y, de esta manera, disminuir sensiblemente las quejas y reclamos.

Tener control sobre los productos no conformes ayuda no sólo a encontrar las causas, sino también a generar las acciones correctivas necesarias para evitar reincidir 
en los mismos errores, lo que conducen a la organización hacia la mejora.

Tener una guía clara sobre el manejo de los productos no conformes ayuda a optimizar el uso de los recursos y, de esta manera, a mejorar la eficiencia de los procesos.

Mediante la inspección de aprobación a cada lote de producto terminado se detectaron unidades defectuosas por debajo del nivel aceptable de calidad. De manera inmediata se procedió a realizar el rechazo y a identificar el lote como producto no conforme. Haber detectado esta eventualidad generó el rechazo del lote compuesto por 2.513 unidades, de las cuales fue necesario reasignar 760. El impacto en el personal del área de empaque fue alto, pues fue el primer lote rechazado en el transcurso del año con cargo asignable al área. A partir de este momento se tuvo conciencia de la efectividad de la inspección por aprobación y del nuevo papel que desempeña el área de calidad.

En otra ocasión, mediante la inspección de aprobación fue posible detectar otro lote con unidades defectuosas. En este caso el lote estaba compuesto por 731 unidades, de las cuales aproximadamente el $40 \%$ tenía fallas de hermeticidad. Al realizar el análisis de causas se encontró una deficiencia importante tanto en el modo de operación de la máquina selladora, como de la máquina en sí misma.

Además del reproceso realizado para eliminar la no conformidad y garantizar la calidad del lote despachado, se realizaron acciones correctivas enfocadas a evitar que el evento se repitiera. Se diseñó una selladora semiautomática que permitiera controlar todas las variables que inciden en la hermeticidad del sellado, como son la temperatura, la presión y el tiempo de contacto. De esta manera se brindó una herramienta más confiable al grupo de trabajo que aumentó su efectividad y les facilitó mejores condiciones laborales.
Otro caso particular de no conformidad de un producto en proceso fue detectado mediante el uso de los gráficos de control. Con ellos se identificó un bache de café tostado, por fuera del rango de color. Esta herramienta además de identificar la no conformidad, brinda información para su tratamiento.

$\mathrm{Al}$ analizar las causas se pudo determinar que un daño de tipo eléctrico impidió que el ventilador de la vasca de enfriamiento funcionara en el momento del descargue de café. La alta temperatura y la falta de ventilación hizo que el proceso de tostión continuara y el color quedó por encima de los límites establecidos. Al detectar esta no conformidad se actúa de acuerdo a lo definido. El analista de calidades realizó las respectivas pruebas en el laboratorio y determinó que el bache no era apto para el producto que inicialmente estaba programado, por lo que fue necesario realizar incorporación a otro producto con características sensoriales más fuertes. El proceso de incorporación fue controlado por el laboratorio para verificar que los perfiles del café inicial y del café al cual se realizó la adición conservaran sus características de acuerdo a lo diseñado.

El uso de estas herramientas ha permitido que el personal de los procesos de tostión, molienda y empaque reciban una retroalimentación grafica y en tiempo real de su desempeño. Observar cuándo y cuánto se desvían de los límites de diseño permite que corrijan los procesos de manera más oportuna.

\section{CONCLUSIONES}

Se logró implementar el procedimiento de control de producto no conforme en la planta torrefactora de café, lo que ha permitido disminuir el número de reclamos recibidos.

Se implementó la inspección de aprobación para los insumos y al producto terminado. La detección de 
lotes defectuosos y su posterior rechazo ha generado gran impacto en los proveedores y en los dueños del proceso productivo.

Se ha impedido el despacho de lotes defectuosos a los centros de distribución, lo que disminuye la posibilidad de futuros reclamos.

Se elaboraron las fichas técnicas de los distintos productos elaborados por la planta torrefactora, lo que permite tener un compendio de las distintas variables a evaluar y de los respectivos criterios de aceptación y rechazo.

La gestión realizada por parte de los especialistas ha generado impacto positivo en la organización.

La implementación de este procedimiento es la etapa inicial de un proceso mayor que busca obtener la certificación bajo los criterios de la norma NTC-ISO 9001:2008.

\section{RECOMENDACIONES}

El haber implementado solamente el numeral 8.3, que hace alusión al control del producto no conforme de la norma ISO 9001:2008, y haber logrado resultados tangibles dentro de la planta torrefactora es un aliciente para continuar con el trabajo de implementación.

Como ya se mencionó, en este momento existen otras prioridades para la organización, las cuales pueden acoplarse sistémicamente con la norma ISO 9001:2008 gracias a la habilidad de los especialistas.

El aprendizaje desarrollado ha sido de gran importancia dentro del ámbito personal y laboral. Experimentar de primera mano que hay modelos de gestión para que una organización mejore su eficacia e incremente los niveles de satisfacción del cliente, y ser gestor de ello, es bastante gratificante.
En el ámbito personal, nuestras vidas y familias son pequeñas organizaciones que deben ser correctamente administradas para alcanzar las metas fijadas. Temas como la misión y la visión marcan nuestro rumbo dentro de todo el abanico de opciones (buenas y malas) que la vida nos ofrece.

\section{REFERENCIAS}

ALMACAFE. Consultado el 15 de septiembre de 2008 en http://www.cafedecolombia.com/quienessomos/almacafe/almacafe.htm

BEATTIE, Phil. Tailoring your roast to the bean. Tea \& Coffee Trade Journal. Consultado el 19 de octubre de 2009 en http://www.entrepreneur.com/ tradejournals/article/198849795.html

CLARKE, John y MACRAE, Robin. Coffee chemistry. Londres: Elsevier Applied Science Publishers. 1985.

CLARKE, John y MACRAE, Robin. Coffee Retead beverages. Londres: Elsevier Applied Science Publishers. 1987.

CLARKE, John y MACRAE, Robin. Roasting and Grinding. En: J. CLARKE y R. MACRAE (Eds). Coffee. London: Elsevier Applied Science Publishers. 1987.

DAVIDS, Keneth. Home Coffee Roasting. Cambridge: St. Martin’s Griffin. 2003.

EPHRAIM, Daniel (2009). The Challenge of grinding coffee for pods and capsules. Tea \& Coffee Trade Journal. Consultado el 19 de octubre de 2009, de http://www.entrepreneur.com/tradejournals/article/198849796.html 
ESCOFFEE. Consultado el 30 de mayo de 2009 en http://www.escoffee.com/es/sobre_blend.php

FEDERACIÓN NACIONAL DE CAFETEROS. Consultado el 15 de septiembre en 2008 de http:// www.cafedecolombia.com/

JURAN, Joseph y BLANTON, Godfrey. Manual de calidad de Juran. Madrid: McGraw Hill. 2001.
KUME, Hotoshi. Herramientas estadísticas básicas para el mejoramiento de la calidad. Bogotá: Norma. 2004.

MONTGOMERY, Douglas. Control estadístico de la calidad. México: Limusa Wiley. 2004.

PROCAFECOL. Consultado el 5 de septiembre de 2008 en http://www.juanvaldezcafe.com/procafecol/

REINA, Mauricio, et al. Juan Valdez, la estrategia detrás de la marca. Bogotá: Ediciones B. 2007. 